Pour obtenir l'arrosage complet minimum, on a fait, sur des calants de $5 \mathrm{~m}$. de longueur, par rapport à des calants de longueur double, toutes choses égales d'ailleurs, une ćconomie du tiers de l'eau d'arrosage. La pesée des récolles nous a démontré que les rendements en fourrage étaient identiques sur toutes les parcelles ayant reçu un arrosage complet.

On réalisera done ure importante économie d'eau, en donnant aux calants une longueur aussi réduite que possible. L'économic est d'autant plus grande que le sol est plus perméable; les calants courts s'imposent surtout dans les lerrains de grande perméabilité.

Conclusions. - De ces recherches il faut conclure que l'arrosage minimum, qui donne toujours les meilleurs résultats culturaux, n'est qu'un arrosage minimum relatif, puisqu'il dépend de la pente, de la végétation, du module et des dimensions des calanls. Il représente toujours un notable excès sur les besoins réels ten eau de la récolte. Il faut chercher à le réduire par le choix rationnel des facteurs dont il est la résultante. De ces facteurs, un seul, le module, est fixé par le règlement du canal. Les autres, qui expriment en réalité l'aménagement du terrain, ne peuvent atre imposés à l'usager. Leur amélioration se traduit tout d'abord par une augmentation des dépenses, mais il en résulte aussi sûrement des récoltes et des bénéfices plus élevés. En attendant que l'agriculteur soit persuadé que, dans son intérêt particulier, comme dans l'intérêt général, il doit s'cfforcer de réeliser des économies d'eau, il serait à désirer que les règlements des canaux d'irrigation l'intéressent directement à ces économies en lui concédant l'eru au volume ct non à la suface arrosée. Cette conclusion s'identifie aveo le principe que MM. Müntz et Faurc ont posé au début de ces longues recherches.

\section{TRAVAUX PUBLICS}

\section{LE RHONE DE MARSEILle A GENÈVE}

Nous avons commencé, le mois passé, la revuc des rapports présentés au IV Congrès de Navigation Intérieure à Nantes gui peuvent intéresser plus particulièrement nos lecteurs. Nous compléterons celte étude en empruntant à $M$. DE DUMas, Direcleur de l'Office des Transports des Chambres de Commerce du Sud-Est, la partie de son rapport sur le Rhône de Marseille à Genève où il montre d'une manière claire et complète que des taxes minimes prélevées sur les marchandises transitées sẹait suffisantes pour rémunérer lc capital cngagé dans cette opération.

Evatuation du trafic probable dU Rhone amélioré. Il paraît intéressant de chercher à évaluer approximativement quelle pourrait être la répercussion sur le trafic du Rhône, des améliorations dont nous venons de rappeler l'utilité, et en particulier de celles que procurerait à la navigation la réalisation du programme préconisé l'an dernier par le Jury du Concours d'avants-projets d'aménagement du Rhòne, organisé par l'Office des Transports des Chambres de Commerce du Sud-Est $\left({ }^{1}\right)$.

Dans 'un remarquablie rapport présenté à la Chambre de Commerce de Iyon, M. le Président Corcner cherchant à déterminer le tráfic probable d'un canal latéral au Rhône après avoir constaté que le trafic de la Seine entre Paris et

(1) Voir à ce sujet, La Houille Blanche. (Mars, Mai, Juin, Juillet 1912). le Havrealleint $58 \%$ du trafic tolal, que celui de la navigation de la Saône prend $29 \%$ du trafic lotal entre Paris et Lyon, tandis que sur lo Bas-Rhòne, celle proportion n'est que de $6,8 \%$ - tient le raisonnement suivant :

"Si le canal latéral existait, quelle proportion de ce trafic de 4082000 tonnes prendrait la Navigalion? Peut-on espérer le laux de $58 \%$ qui existe sur la Seine, ou ne faut-il Labler que sur celui de $29 \%$ que la navigation prend de Paris à Lyon ? Il faut remarquer que le tnafic de la Seine aboutit à un grand port maritime, le Havre, situation comparable à ce que serait Marseille pour le Irafic MarseilleLyon. D'un autre còté, si on examine de quoi se compose le lrafic, on voit que $40 \%$ environ est constitué par les combustibles minéraux. Dans la vallée du Rhône, le trafic de la navigation en combustible n'est que de $3 \%$ du tralic tolal de la navigation. Mais celte situation lient à l'absence de ports de raccordement bien oulillés à Givors et à l'Ardoisc, au débouché des bassins houillens de la loire t' d'Alais, et plus encore peut-être aux tarifs élevés du chemin de fer dic la mine au Rhòne. Cette situation changerait-elle avec le canal latéral ?

"On peut espéner qu'on établirait alors des ports de raccordement ; mais rien ne prouve qu'on obliendrail un abaissement de tarifs de la mine au canal. On peut penser toutefois que le seul abaissement du fret sur le Rhône cnlèverait une partie du trafic de la houille au chemin de fer. Le tonnage de ce trafic, sur le réseau P.-L.-M., a été en rgo5 de 8 I 74 ooo tonnes sur 27757 ooo tonnes de trafic total, soit $29 \%$. Pour la navigation sur la Saône, on trouve de méme que les combustibles forment $29 \%$ du trafic. On peul donc penser qu'il en serail de même pour le Rhòne, el que même la houille anglaise arrivant par le port de Marscille donnerait un chiffre supérieur.

"Pour toutes ces considérations, on peut admettre que, dans le partage qui se produira entre lo chemin de fer et la navigation, cette dernière prendra une proportion intermédiaire entre les $58 \%$ de la Seine, et les $29 \%$ de la Saône, soit la moyenne de $43 \%$.

"Dans son rapport, à l'appui de l'avant-projet du canal latéral, M. Armand, ingénieur en chef de la navigation du Rhône, adopte le cocfficient de 38 \% qu'avait indiqué en Igor, M. Guérard, ingénicur en chef des Ponts el Chaussécs, auteur du projet du canal de Marscille au Rhône, et qui est ]a part de la navigation dans le trafic tolal de Paris à Nancy. Mais, comme nous lic disions plus haut, l'exemple de Nancy est moins probant que celui de la Seine, car le trafic de Paris à Nancy n'aboutit pas à un port de mer. Si nous voulions prendre un exemple à l'étranger, nous voyons qu'à Hambourg en 1898 , la navigation intérieure a pris $66 \%$ du trafic total. Nous croyons done pouvoir adopler le coefficient de $43^{\circ} \%$, comme devant s'établir à la longue.

"Si nous appliquons ce coeflicient de $43 \%$ au trafic total de Lyon à la Médilerranće de i 905 , que nous avons vu être de 4082 ooo tonnes, nous arrivons au chiffre de 1755000 tonnes. M. Guérard arrivait, en rgor, au chiffre très voisin de I 600 ooo tonnes, par le raisonnement suivant : Le trafic total de Lyon à Marseille était à celle époque, de 2 go8 ooo tonnes, dont 2667 ooo tonnes par le chemin de fer. Avec lo coefficient de $38 \%$, la navigation doit enlever un million I 05 ooo tonnes. Mais le chemin de fer défendra son tonnage et ce n'est que lorsque le tonnage total aura atteint 4 millions 300 ooo tonnes que l'équilibre s'établira avec :

$62 \%$ pour le chemin de fer ou 2666 ooo tonnes.

$38 \%$ pour la navigation ou I 634 ooo tonnes.

"En raisonnant de ]a même façon, avec les chiffres de la 
stalislique de 1905, que nous avons cités, nous dirions que le chemin de fer défendra son tomnage de 3802 ooo lonnes, ot que ce n'est que lorsque le tonnage aura atteint 6670 ooo tonnes que le trafic se partagera entre : le chemin de fer prenant $57 \%$ ou 3802 ooo tonnes, et la navigation prenant $43 \%$ ou 2868 ooo tonnes.

"Le tonnage total de 4300 ooo tonnes, prévu par M. Guérard en Igor, a ćté presque attcint dès I 905 \%.

On pourrait done croire que le tonnage de 6600 ooo tonnes serait atteint quelques annécs après l'ouverture du canal. Admettons un chiffre intermédiaire entre ce tonnage de 2868000 tonnes et le chiffre de $x 600$ ooo tomnes calculé par M. Guérard, et accepté par M. Armand, el raisonnons sur 2300 ooo tonnes.

Ce raisonnement est encore applicable dans le cas prósent. M. Coignet supposait pour établir ce chiffre que le fret sur lc canal étail abaissé à o fr. or? par tonne kilométrique; or. comme nous l'apprend le rapporl de M. ne MAS, le prix du fret sur le Rhòne amélioré lui serait comparable.

Nous pensons que le chiffre de 2300 ooo tonnes ne serait qu'un minimum rapidement dépassé. II résulte, en effet, du seul partage de trafic entre le chemin de fer P.-L.-M. et le Rhône, tel qu'il existe actuellement, el en no tenant pas compte du trafic nouveau que l'élévation des tarifs P.-L.-M. no permet pas d'effectuer et qui devicndrait possible après l'abaissement des prix.

Il est, d'autre part, certain que l'amélioration des voies d'eau aboutissant au Rhone, ainsi que la mise co communication directe du fleuve avec Marseille ct Celte par des canaux sur lesquels pourraient circuler les barques du Rhône, el la création d'un grand port de commerce à Lyon, constitueraient un faisceau nouveau de circonstances favorables à l'extension d'u trafic.

Enfin, et sans avoir la prétention de donner une évalua tion même approximalive de l'accroissement des transporls qui pourrait en résulter, il est hors de doute que le développement de l'industrie que ferait nattre les facilités données aux transports tant dans la vallée du Rhóne que sur les canaux d'Arles à Marseille et de ceinture de Lyon, provoquerait sur le chemin de fer et sur la voic d'eau un accroissement continu dit trafic.

Maur-Ruone. - Les travaux d'amélioration cxéculés dans la première section du Rhône (déviation du Sault) n'ont eu jusqu'à présent que des effets locaux, el sont sans influcnce sur l'état général de la navigabilité.

Le trafic du Ilaut-Rhône ne pourra sc aćvelopper d'une façon normale qu'à parlir du moment où la navigation sera possible dans de bonnes conditions entre Lyon et Genève: Or, la construction de l'usine hydro-électricue projetée à Génissial serait un premicr pas important dans cetle voie. Le barrage sur le Rhône constituerait, en effet, 'un bief navigable de 23 kilomètres de longueur de Génissiat à Chancy, à Ia frontière suisse. Il suffirail alors de construire un ascenscur à Génissiat et des échuses aux barrages des usines établies par la ville de Genève à la Plaine-Chèvres et la Coulouvrenière, pour que le Rhòne deviénne naviguable entre Génissiat et le lac Léman. La traversée de Genève dont le tirant d'air des ponts est trop réduit pourrait être évitée par un canal de faible longueur, contoirnant la ville.

Le nionlant des travaux à exécutcr entre Génissiat et Genève poul rendre ces communications possibles est évalué par les auteurs du projet à 20 millions, y compris l'ascenseur de Gónissiat. Enfin les travaux de régularisation à effectuer dans la partic inféricure du Maut-Rhône sont estimés à 3. millions.
Le Conseil général des Ponts et Chaussées appelé à donner son avis sur l'opportunité d'entreprendre les travaux nécessaires pour assurer les relations par eau entre Lyon of Genève, a formulć les conclusions suivantes :

"La création d'une voie navigable aboutissant à Genève n'offre aucun intérêt pour la France. Il n'y a aucun trafic international appréciable à prévoir ni dans la direction de Strasbourg, ni dans celle de Bale ou de Genève, pour des voies navigables a ouvrir dans la vallée de la Loirc ou dans celle du Rhòne el en conséquence, il n'y a aucun travail à proposer pour atteindre ce résultat $"$.

Cetle appréciation a soulevé de la part du Commerce de vives protestations el nous ne pouvons mieux faire pour réfuler. los arguments du Conseil général des Ponts ct Chaussćes que de reproduire en partie la réponse faite à ces conclusions par la Chambre de Commerce de Lyon. (Rapport du 6 janvier $\mathrm{sg}$ ro, de son Président, M. Coignet.)

"Le Conseil gónéral des Ponts et Chaussces pour repousser celle voie fait un raisonnement analogue à cèlui qu'il a fait pour condamner le canal du Rhône au Rhin. Il considere cetle voie comme faisant partie de la grande voie de Marseille-Genève-Bâle-Strasbourg, et il n'a pas de peine à montrer que. Ia distance de Rotlerdam à Strasbourg étant de 698 kilomètres et celle de Marseille à Strasbourg do $9^{\circ 3}$ kilomètres par le Rhône au Rhin ou 992 kilomètres par Genève, avec beaucoup plus de difficultés que sur le Rhin; la voie Marseille-Strasbourg ne peut lutler. contre celle do Rotterdam.Strasbourg.

"Pour Bâle, la comparaison est déjà moins défavorable. Il y a 830 kilomètres de Rotterdam à Bâle, il y a de Marseille à Bâle 83 r kilomètres par le Rhòne au Rhin et 873 kilomètres par Genève et los lacs suisses.

" Mais sans nous arrêter aux marchandises qui onl leur marché commercial à Marscille et qui, malgré une différence de frais de transporl, utiliscront cette voie, sans parler des marchandises produiles par l'industrje marseillaise et qui pourraient lutter contre la concurrence de l'industrie allemande, nous rappellerons encore que la distance moyenne des transports par eau en France étant seulement de r 54 kilomètres, c'est par sections de longueur du même ordre qu'il faut étudier "l'utilité d'une voie navigable ".

"La voie de Lyon à Genève aura 2 ro kilomètres. Fn elle-même, quel trafic aura-t-elle ? En rgo6, le trafic par chemin de fer de la ligne Lyon-Genève et ide l'embranchement Mâcon-Bourg a été de 27 r ooo ooo de tonnes kilométriques. Lorsque lo Rhône sera rendu navigable de Lyon à Genève dans de bonnes conditions, on peut admettre que, comme de Lyon à Paris, il cnlèvera $29 \%$ du trafic au chemin de fer, soit 78590 ooo tonnes kilométriques, ce qui représente un tonnage moyen de 374 ooo tonnes, c'est-à-dire un peu moins que le tonnage de la Saône de Lyon à Saint-Jeande-Losne qui est de 456 ooo tonnes.

"En particulier, nous signalerons le débouché qu'auront sur Genève et les villes du lac Léman, les malériaux de construction (pierres de taille, chaux et ciments) produits dans notre région. Actuellement ces matériaux qui viennent ì Lyon par la batellerie du Haut-Rhône ont atteint en rgo8 un tonnage de 167 ooo tonnes. Un tomnage important s'établirail done pour la Suisse. Le coût du transport par chemin do fer de Lyon à Genève pour les grosses marchandises cst, par tonne, de $7 \mathrm{fr} .7^{5}$ à la sixième série et de 6 . fr. 20 au barème $\mathrm{E}$, soit en moyenne 6 fr. 92. Sur le Rhône éclusés. le fret doit tomber à o fr. ox 6 , soit 3 fr. 36 , pour 2 to kilo mètres de Lyon à Genc̀ve. L'économie sera donc de 3 fr. 36 par tonne, soit i $33 \mathrm{x}$ ooo francs, ce qui à $3 \%$ représente 
un capilal de 44 millions. Le Conseil général des Ponts et Chaussées évalue à 54 millions les dépenses à faire sur le Haut-Rhóne pour la navigation (22 millions du canal de savières à la frontière suisse, et 32 millions de Lyon au canal de Savières). Il suffirail donc que les usines hydrauliques à ćtablir fournissent une subvention de to millions, pour que l'ouvre fut cntièrement équilibrée.

"D'un autre cốé, le lac Léman ofrrira un débouché fort intéressant pour les chalands du Rhône. Ce lac a, en effel, une longueur de $7 \mathrm{r}$ kilomètres sur sa rive sud et un développement de côtes de 152 kilomètres. A Lausanne, Jes chalands du Rhône pourront transborder dans la direction de ja Suisse centrale et, à Villeneuve, dans celle du Simplon. Co sera là une source importante de trafic.

"Si, plus tard, la Suisse exécule le réseau qu'elle projells, reliant le lac Léman au lac de Neufchâtel et au Rhin, ce trafic en recevra un nouvel accroissement. Si, pour Bâle même, comme nous l'axons montré, ce trafic passe en parlì ou même entièrement par lo canal du Rhồne au Rhin ambioré, il restera un trafic important pour tout l'intéricur de la Suisse. Mais ces perspeclives d'extension ne sont nullemenl néccssaires pour légitimor la voie de Lyon à Genève; celle voic est utile en clle-même pour tous los échanges entre la vallée de la Saône et Lyon el le bassin du Léman. Mais si le frot de Marsoille à Lyon étail abaissé. à francs la tonne, par le canal latéral, celle voie prendrait une importance notrvelle. En effet, si le transport du blé conte actuellement I3 fr. 60 la tonne par fer, de Marseille à Genève, il ne coûterait plus que $7 \mathrm{fr}$. 36 dans le cas de l'exécution entièrc du canal.

"Il est donc corlain que la pénétration du blé comme de toutc autre marchandise débarquée à Marseille irait beaucoup plus loin dans la Suisse qu'actuellement.

"Nous nous élevons done avec énergie contre cette asscrtion du Conseil général des Ponts et Chaussées, que la voie navigable de Lyon à Génève n'offre aucun intérêt pour la linance $n$.

Comté Franco-Surse du Haut-Ruone. - L'aménagement đu Haut-Rhône est donc une question d'inlérêt général qu’il est essentiel de poursuivre.

L'entente des Gouvernements suisse et français est indispensable pour que les travaux en France et cn Suisse soient conduits parallèlement, et en particulier pour donner aux écluses les mêmes dimensions dans les deux pays.

Pour préparer celte entente, la Chambre de Commerce française de Genève a eu l'excellente idée de former un Comité compétent de personnalités suisses et françaises. Le "Comité Franco-Suisse du Haut-Rhônc " ainsi constitué, soccupe activement de cette question.

Etat actuel de la question. - L'autorisation de la captation de la force motrice sur le Haut-Rhône ne peut être accordée que par une loi.

Le Ministère des Travaux publics a nommé une Commission de géologues présidés par M. Termier, inspecteur génémil des Mines, pour étudier les emplacements proposés pour les barrages par les divers demandeurs en concession. Dès que cette Commission aura donné son avis, le projet de loi pour octroyer la concession sera sans doute déposé devant le Parlement.

Mais il est un point sur lequel nous jugeons utile d'attirer l'allention du Congrès. Le Gouvernement qui n'alloue aucune subvention aux concessionnaires se réserve, dans le cahier des charges, un droit de partage des bénéfices. II serait désirable que les sommes revenant ainsi à l'Etat soient consacrées à l'amélioration de la navigation du Haut-Rhône.
Cel abandon des bénélices à la région qui les provoque ne serait que la juste contre-partie de la contribution des corps locaux dans les travaux de navigation que l'Etat ne veut plus entreprendre sans leur concours. Le Congrès pourrait formuler un vou lendant à ce que les bénéfices revenant à l'Etat soient alloués - ponr être employćs aux travaux du Haut-Rhône - à l'office National de la Navigation investi de l'aulonomic financière, qui vient d'etre créc au Minislère dos Travaux publics et donl le xôle principal est " de rechercher tous les moyens propres à développer la navigation, do provoquer $\mathrm{el}$, au besoin, de prendre toules mesures tendant à ameliorer l'exploitalion des roies navigables $n$.

D'autre part, il serait de toute nécessité d'imposer au concessionnaire de l'usine hydro-électrique l'obligation de fournir grafuitement l'énergic nécessaire pour lo passage des bateaux d'un bief a l'autre.

Conclusion. - En résumé, nous proposons au Congrès d'émetire les voux suivants :

$x^{\circ}$ Pour lamélioxation du Rhone en aval de Lyon :

Que le Gouvernement constitue un service chargé de l'ćlude complète du programme fléconisé par le Jury du concours d'avant-projets d'aménagement du Rhóne organisé par l'office des Transporls des Chambres de Commerce du Sud-Est.

$2^{\circ}$ Pour la navigalion du Haul-Rhône :

Que la concession des forces motrices du Haut-Rhône ne soil accordée que sous réserve de l'obligation pour les concessionnaires de fournir, à titre gratuit, l'énergie nécessaire pour assurer le passage d'un bief à l'autre cl l'éclairage des divers services.

Que la part des bénéfices de l'entreprise revcnant à l'Elat, soit remise à l'Office National de la Navigation, pour être affectée à l'amélioration de la navigation du Haut-Rhône.

$3^{\circ}$ Ports de raccordement

Qu'il soit créć des ports de racordement échelonnés le long du Rhône, que ceux cxistant actuellement soient améliorés et que leurs larifs soient révisés.

$4^{\circ}$ Ecluses de la Petite-Saóne:

Que le projet d'agrandissement des écluses de. Ia PeliteSaône soit renvoyé pour étude définitive au Service de la Navigation du Rhóne et de la Saone.

$5^{\circ}$ Canal du Rhône au Rhin :

En présence de l'attitude du Gouvornemenl allemand qui a manifesté son désir d'arriver à une solution sur son territoire, que la question de mise au gabarit normal soil reprise ef mise au point sur le territoire français.

$6^{\circ}$ Canal de Roanne à Saint-Etienne :

Que le canal de Roanne à Saint-Ftienne soil compris dans le nouveau programme des travaux publics que l'on examine à la Commission du Sénal, en réduisant au minimum la subvention à fournir par les particuliers.

$7^{\circ}$ Que des études soient entreprises pour augmenter Jc tirant d'air des ponts du canal du Rhône à Cette, afin do permettre l'accès de cette voie d'eau aux barques du Rhône.

\section{COMMISSION EXTRAPARLEMENTAIRE DES GRANDS TRAVACX DE NAVIGATION}

Nous extrayons du bulletin trimestriel de La Navigalion Intérieure paru en avril rgr3 une étude qui résume les travaux entrepris par la Commission Extraparlementaire des Grands Travaux de Navigation, et montre quelle est l'opinien de cetle commission et quels sont les projets qu'ello soutiendra devant le Parlement. 
Dans des discussions qui se sont tout récemment déroulées au sein de la Commission extraparlemenlaire des iravaux publics, il a été dit, et non par les premiers venus, yu'un des principaux rôles joués par les voies navigables avail consisté à provoquer l'abaissement des tarifs de chemins de fer.

A cette appréciation, il a été fait, en une ou plusieurs fois, les réponses suivantes qui paraissent avoir représenté l'opinion moyenne de la Commission.

Ceux qui ont créé ou contribué à créer les voies d'eau ont-ils pensé leur faire jouer ce rôle ? On cn peut douter. En tout cas, il ne peut plus être de mise, pour les raisons suivantes :

I Parce que dans l'ćtat de nos finances, on ne peut plus recourir à des procédés aussi détơurnés et aussi coûteux.

$2^{\circ}$ Parce que les chemins de fer, à moins d'être fous, no peuvent plus, en face des charges maltiples dont ils sont écrasés, abaisser leurs tarifs.

$3^{\circ}$ Parce qu'en supposant qu'ils conçoivent des projets aussi aventureux et tentent de concurrencer la batellerie à coup de rabais, ils en sont empêchés par la doctrine du Comité consultatif des chemins de fer qui ne tolère pour ainsi dire plus de prix fermes.

Les voies d'eau ne doivent donc être construites que si elles rendent des services directs, c'est-à-dire si elles permettent de réaliser des prix de transport notablement infćricurs à ccux de la voie ferrée. En effet, elles sont infćrieures à celle-ci par nombre de points et, d'autre part, il est contre-indiqué de faire la dépense d'un nouvel outil s'il n'est pas plus avantageux que celui que l'on possède.

Peut-on lui faire réaliser ce desideratum, non seulement cn apparence, mais en réalité, c'est-à-dire en portant au débit de la voie d'cau non seulement le coût de son entretien, mais la charge du capital consacré à son établisšement? Oui, mais à deux conditions :

$\mathrm{I}^{\circ}$ Qu'elle soit établie avec une ampleur suffisante pour que le coût du transport y puisse être très bas.

$2^{\circ}$ Que sa fréquentation puisse être assez considérable pour que les taxes d'usage, tout en étant modiques et en ne créant pas par conséquent un obstacle à cette fréquentalion, produisent suffisamment pour payer les charges de capital et d'entretien.

Les hommes renseignés et édifiés, qui composent la Commission extraparlementaire, savent en effet, tous ou presque tous, que l'on pose parlout en axiome en Europe comme dans le Nouveau-Monde, que l'on ne doit plus créer de voies nouvelles à petit gabarit. Cet axiome a été consacré une fois de plus au Congrès international de navigation qui s'est tenu en rgra à Philadelphie.

Certains esprits avaient pu croire la transformation de la traction susceptible de réaliser une diminution suffisante dans le coût des transports par eau pour que l'augmentation du gabaril de 1879 ne s'imposât pas; mais il n'en est pas ainsi. On ne saurait nier que, notamment, la traction électrique, qui ne fait que débuter et qui n'est pas encore toul à fait au point, soit susceptible de réaliser d'appréciables avantages, mais ceux-ci ne sont pas d'un ordre de grandeur lel que lés économies finales que l'on poursuit puissent être réalisées par ce seul moyen.

Saisie des propositions de la Commission Interministépielle relatives à l'affectation des ressources annuelles du budget, considérées comme normales, à un ensemble de grands travaux évalué, tant pour les ports maritimes que pour les voies de navigation intéricure, à I $100000000 \mathrm{dc}$ franes, la Commission Extraparlementaire a estimé qu’il convenait de faire une distinction dans les travaux énumérés et d'en dresser une double liste. Dans la première figureraient les travaux dont la partic de dépense qui resle la charge de l'Etat continuerail à être payće directement sur les ressources annuelles du budget; dans la sccondé, on inscrirait les travaux dont la dépense à la charge de l'Etat serait payée au moyen d'annuités.

La Commission Interministérielle, se conformant à la mission qui lui élait confiée, a procédé à ce travail de révisioli. Elle s'est inspirée des considérations suivanteś : Elle a admis que l'on devait continuer à payer directement sur les ressources annuelles du budget les travaux qui, aussi bien dans les ports que sur les voies de navigation intérieure, ne conslituent que des améliorations successives et pour ainsi dire continues. Ces travaux, réalisés au fur et à mesure des besoins et d'une utilisation immédiate et complètc dès lcur achèvement, ne peuvent être considérés comme des créations nouvelles dont les générations futures auront à bénéficicr et dont elles doivent, comme conséquence, supporter la charge. Elle a, au contraire, rangé dans cette dernière catégorie, tous les grands travaux qui ont pour but de préparer l'avenir, de conslituer le cadre dans lequel viendront se placer les améliorations successives. On peut citer dans cét ordre d'idées le canal maritime de Grattequina à Bordeaux sur lequel s'ouvriront successivement, suivant les besoins, une série de darses ; - la grande digue du Havre, à l'abr: de laquelle un nouveau port pourra se développer ; - les approfondissements de la Seine, de la Loire, de la Girondc; dont les navires de tirant d'eau croissant, profiteront dans l'avenir.

Des principes analogues ont été appliqués pour l'établissement de la double liste des voies navigables.

Les tableaux ci-annexés indiquent la répartition qui a été faite par la Commission Interministérielle à la suite d'un examen détaillé des différentes opérations énumérées.

Il en résulte que les dépenses à payer directement sur les ressources annuelles du budget s'élèvent, pour les voies de navigation intérieure, à .............

En calculant sur une durée d'exécution de r/4 ans et demi pour les voies de navigation, on trouve que les dépenses à imputer annuellement sur les ressources normales du budget sont, pour les voies de navigation intérieure, environ........

Les crédits considérés comme normaux étant pour ces mêmes voies .......... il resterait disponible, pour le service des annuités $\ldots \ldots \ldots \ldots \ldots \ldots \ldots \ldots$

Si l'on suppose des emprunts fails au taux de $4 \%$ remboursables en 56 ans, l'annuité est de $4,5 \%$. Il serait donc possible de gager, pour les voies de navigalion intéricure, un emprunt de .......... 278 ooo ooo "

En ce qui concerne les voies de navigation intérieure, une somme de ró' millions est à réserver, comme il est indiqué pour l'exécution de la parlie des travaux en cours qui serait payé au moyen d'annuité; il reste alors disponible une somme de I 7 I millions pour des opérations nouvelles à entreprendre.

Sur cette somme, on propose de prévoir dès maintenant l'imputation des trava'ux ci-après :

Seine à Paris et dans la banlieue. - Travaux de défense contre les inondations, mais concus dans le but d'améliorêr? 
en même temps la navigation, 68 ooo ooo de francs, dont 3:

Canal de Berry. - Mise au gabaril normal, 6 I 200 ooo francs, dont 48000000 francs à la charge de l'Etat.

Le surplus, c'est-à-dire 9r ooo ooo de franes environ, serait employé soit à certaines des entreprises dont. les études so poursuivent, et dont la nomenclature ainsi que la situation. seront présentées à la Commission dans sa prochaine réunion, soit par l'cmploi de l'annuité correspondante, à la réduction du délai d'exécution des travaux qui continueront i être payés sur les ressources ordinaires.

Au point de vue de lá procédure à adopter pour réaliser res combinaisons, le système le plus simple consiste à demander les avances nécessaires aux Chambres de Commerce, Départements, Villes ou Etablissements publics intéressés, in leur assurant le remboursement de ces avances au moyen d'annuités calculées comme il vient d'être indiqué. Dans cos conditions, ces établissements pourront réaliser leurs rmprunts à un taux qui ne différera guère de celui que l'Etat obtiendrait lui-même, sans que celui-ci soit obligé de recourir à dès emprunts directs.

Il scrait d'ailleurs entendu que les avances ne seraient sollicitées qu'au fur et à mes'ure des besoins reconnus et que le remboursement anticipé serait toujours possible.

Si la combinaison cnvisagée est adoptée, il y aura lieu tout d'abord de solliciter du Parlement l'autorisation de de. mander aux personnes morales intéressées des avances remboursables par annuités, calculécs aù taux d'intérêt maxinum de $4 \%$ et pour une durée maxima de 56 ans, jusqu'à concurrence, pour les travaux en cours, de r 10 ooo ooo de francs pour les ports maritimes, et de 104 ooo ooo de francs pour les voies de navigation intérieure; les autres autorisations seraient sollicitées en même temps que la déclaration l'ulilité publique de chaque opération.

\section{LE HALAGE ÉLECTRIQUE SUR LES CANAUX}

Comment pouvons-nous améliorer el rendre rémunẻraleur le trafic sur les canaux intérieurs ? Sans aucun doute, lạ transformation des canaux à faible section mouillée en canaux larges et profonds cst la solution la meilleure, mais combien coûteuse. Ne pourrait-on améliorer los canaux existants, accroître leur capacité de trafic en employant les ressources de l'industrie moderne ? C'est la tâche que s'est imposée à la Compagnie Générale Electrique de Nancy et elle insiste sur la possibilité et les avantages que présente le hâlage électrique des bateaux.

Nous reproduisons ci-après une étude sur ce sujet.

Le développement de l'industrie a profondément modifié, dans ces dernières années, les conditions des transports par cau. Non seulement le trafic cst considérablement augmenlé, mais encore on exige impérativement la diminution des délais de transport. Il s'agit donc, non pas seulement le multiplier les voies navigables, mais plutôt d'organiser des halages mécaniques, ce qui permet, à la fois, d'accélérer l'allure des bateaux et d'augmenter le trafic sur les canaux existants.

En vue de réaliser le problème di halage mécanique, on a fait de nombreux essais qui n'ont pas toujours étć cour'onnés de succès.

C'est ainsi que le long du canal de Teltow, construit pour éviter aux bateaux utilisant la Sprée, la traversée de Berlin, on a installé, sur chaque berge, une voic ferrée, et le halage se fait par locomotives électriques. Cette solutioni a ćté rendue possible par ce fait que le canal de Tellow a élé consrruit spécialement en vue de son application, el son prix de revient est tellement élevé qu'elle ne peut se justificr que par le trafic extrêmement intense el toul à fait d'exception de ce canal.

Sur les canaux du Nord on essaya d'abord des tricycle; tracteurs sur berges; ils furent abandonnés en raison de leur inefficacité. On appliqua la traction par locomotives, mais en n'équipant qu'une berge avec voie simple, perdant ainsi en grande partie les avantages du syslème de Teltow. Malgré cela, lo prix de revient reste encore lrès élevé et il est nécessaire d'être assuré d'un trafic important pour ru'une installation semblable soit rémunératrjce.

Le système par tracteur's sur berges présente, non seulement l'inconvénient grave d'êlre d'une applicalion onćreuse, mais, dans la plupart des cas, l'établissement de voies est rendu très difficile par les coudes brusques du canal, la présence de ponts, de traversées de rivières el d'ouvrages d'art de toutes sortes. L'adaptation d'un tel système entraîne donc des dépenses considérables, hors de proportion avec le résultat oblen'u.

Le haleur flottant ne peut guère être pris en considération car il entraîne le double ćclusage, ce qui, dans l'état acluel des canaux, limile son emploi aux canaux sans écluses.

Ces différents systèmes ne peuvent donc êlro considérés que comme des solutions particulières, justifiées soit par un trafic considérable, soit par des conditions locales exceptionnellement favorables.

Pour donner au problème une solulion générale, il fallail trouver un système capable de s'adapler à tous les canaux, simple, robuste et sûr, peut-être moins complet techniqucment que ceux que nous venons de rappeler, mais moins coûteux comme premier établissement, étant bien établi que la vitalilé d'une entreprise de halage électrique dépendrait avant tout du capital engagé. C'est le problème que s'est posé la Compagnic Générale Elcctrique de Nancy et nous nous proposons, dans la description qui va suivre, de montrer comment clle l'a résolu.

Le principe consiste à haler les bateaux par câbles sans fin longeant la berge. Ces câbles ont tous une longueur uniforme d'environ 450 mètres al chacun d'eux est actionné par un cabestan électrique.

Si l'on suit le bord d'u canal, on rencontre d'abord un cable sans fin, dont les deux brins cheminent en sens inverse et ont environ chacun 450 mètres de longucur, puis un espace libre de 50 mètres environ, un nouveau câble sans fin et ainsi de suite.

Chaque cabestan, commandé par un moteur électrique, peut être mis en route ou arrêté par deux interrupleurs spéciaux, placés aux deux extrémités du câble.

Le halage se comprend alors sans difficulté. Supposons un bateau isolé sur le canal. Il est accompagné par un conducteur (analogue au charreticr ou commis actucl) qui est chargé de manœuvrer les cabestans et la corde de halage. Le conducteur accroche l'amarre au câble sans fin et le bateau est remorqué sur $45 \mathrm{c}$ mètres de longueur, en partant d'une vitesse initiale nulle, pour être accéléréc graduellement jusqu'à l'allure de 3 kilomètres à l'heure, ceci par le simple effet du moteur électrique du cabestan, qui, grâce ì une combinaison spéciale, réalisc le démarrage avec un couple très puissant el une accélération automatique de vitesse sans aucun appareil auxiliairc. Quand le point d'arrimage vient à l'cxtrémité du câble sans fin, le conducteur 
arrête le cabestan, décroche l'amarro et, suivant au pas le: bateau qui, par sa vitesse acquise, continue a avancer, vient accrocher l'amarre au câble sans fin suivant, dont il met le cabestan en route. le batcau est ansi halé successivement par chaque cabestan, coux-ci fonctionnant unjqutement pendant le temps que met lo batcau à parcourir la longueur du câble.

Pour hâler los bateaux dans l'un ou l'autre sens, il suffit alors do fixer l'amarre à l'un ou à l'autre brin des câbles sans fin.

$\mathrm{Si}$ nous avons maintenant'à faire à plusicurs baleaux cheminant simultanćment dans le même sens on devra s'arranger pour que chaque cabestan remorque un seul bateau; ceuxci devront donc se suivre à un intervalle rógulicr de 500 mèlres ou

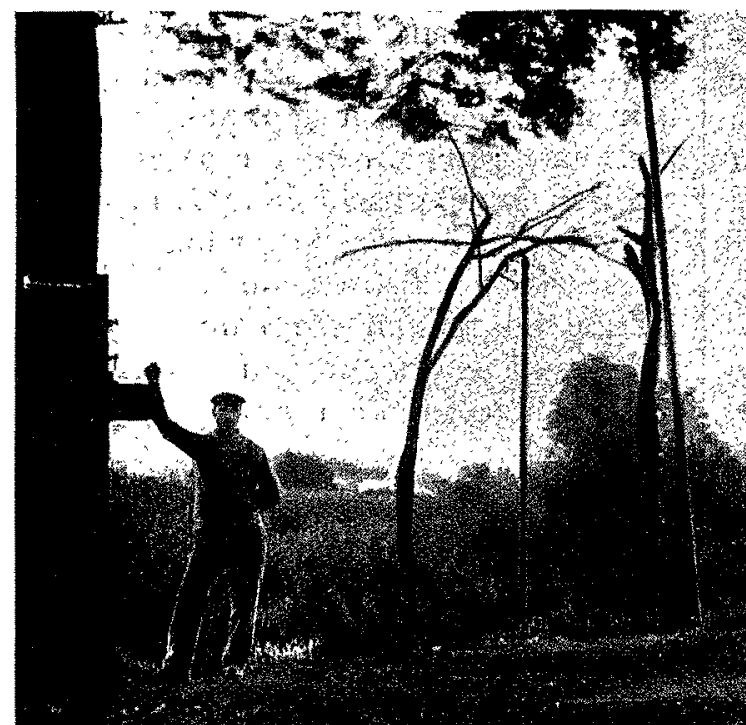

teur manœurre l'interrupteur de mise en marche et d'arrêt au centre, le cabestan; à droite, le long de la berge, les poulies portouses et le câble.

Cherchons à nous rendre comple, dans ces conditions, du débit du canal par jour, c'est-à-dire du nombre de bateaux

mulliple cntier de 500 mètres (distance de deux cabestans).

$\mathrm{Si}$, enfin, nous nous plaçons dans le cas le plus général de bateaux circulant dans les deux sens, un cabestan devra toujours ne remorquer qu'un scul balcau; le débit maximum du canal sera donc oblenu quand chaque cabestan

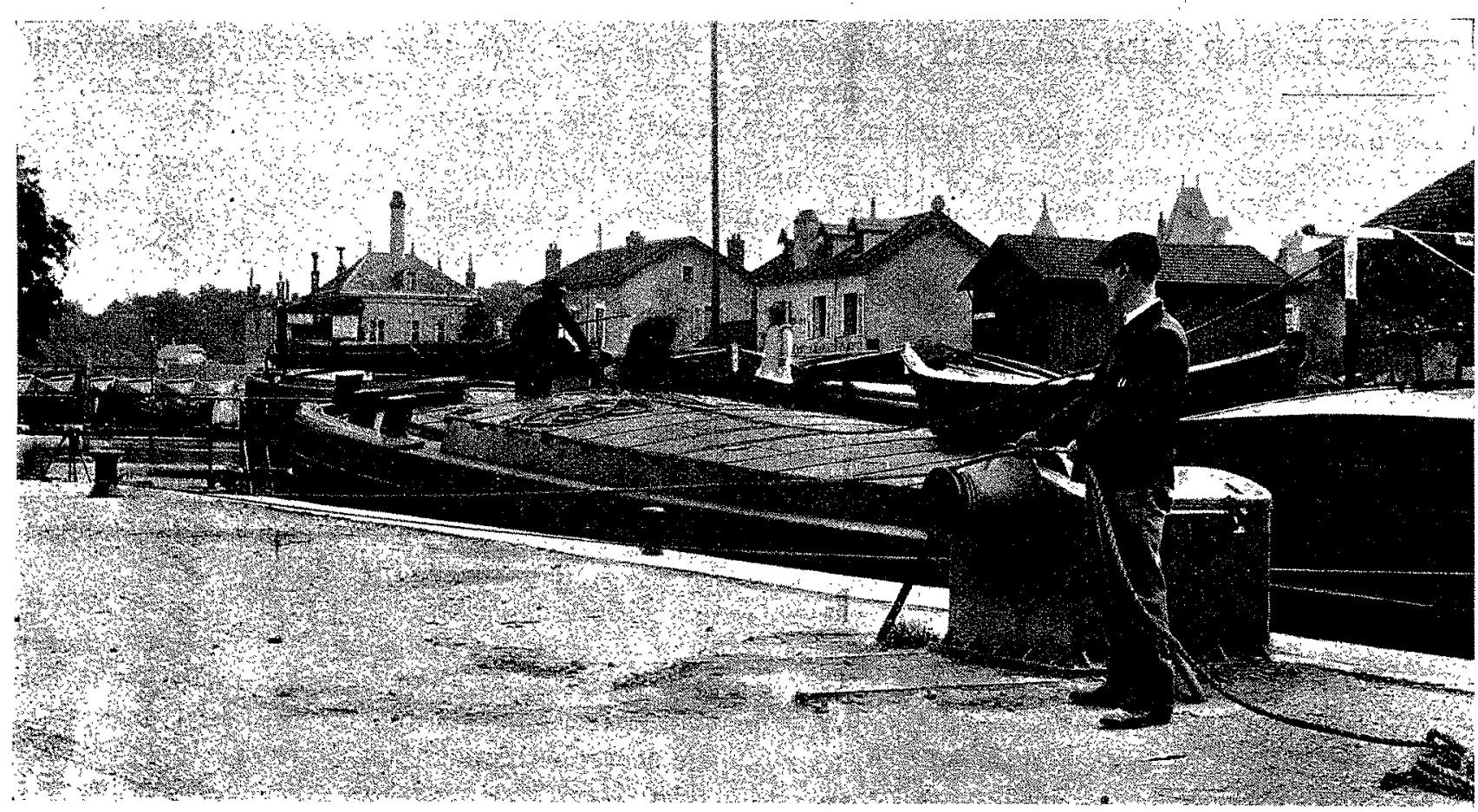

FIG. 2, - DISIOSITIF D'ECLUSAGE EIECTRIOUE.

12 heures de travail.

Le premier élément à fixer est la vitesse des bateaux. Or, l'expérience montre qu'étant donnés la section des canaux, la forme des bateaux et leur tonnage moyen, il n'est guère possible de dépasser la vitesse de $3 \mathrm{~km} .5$ à l'heure. Au-delà, la puissance absorbéc croît considćrablement, pour une augmentation de vitesse peu sensible, et le remous produit devient. très important, surlout au croisement de deux bateaux chargés.

Admettons dono comme vitesse moyenne des bateaux la vitesse de 3 kms à l'heure. Puis que les bateaux marchant dans le même sens sont à une distance l'un de

halcra successivement deux bataux cheminant on sens contraire. La distance entre deux bateaux circulanb dans Je même sens sera donc, cette fois, de $x$ kilomètre,

I'installation telle que décrite ci-dessus iest représentée dans son cnsemble par la ligure ' . A gauche, le conduc- l'autre de I km., il en passera, en un point, six par heure (trois dans chaque sens), soit, dans les deux sens, $7^{2}$ par jour de 12 heures, ce qui correspond à uin trafic très intense.

Il est d'ailleurs clair que pour augmenter le débit, il suffrail de diminuer la longueur de chaque câble. Avec des 
cabestans situés à 333 mètres l'un de l'autre, la distance n'mlre deux bateaux conséculifs n'est plus que de 666 mètres. Il passe en un point 9 baleaux par houre, soit ros par jour.

En pralique, des tralics de celle intensité ne sont guère possibles, ce à cause des écluses. Avec les procédés achels la durée d'éclusage d'un bateau est comprise entre 20 et 30 minules ; une écluse ne peul done laisser passer au maximum que hois bateaux i l'heure.

De ceci il résulto que pour augmenter la capacilé de débit d'un canal, il faut impéralivement réduive les durées d'écl us a ge. Or, cetle dernière opération se compose de deux parties: la manceuvre des rames (re $\mathrm{m}$ plissage ou vidange de l'écluse) et la

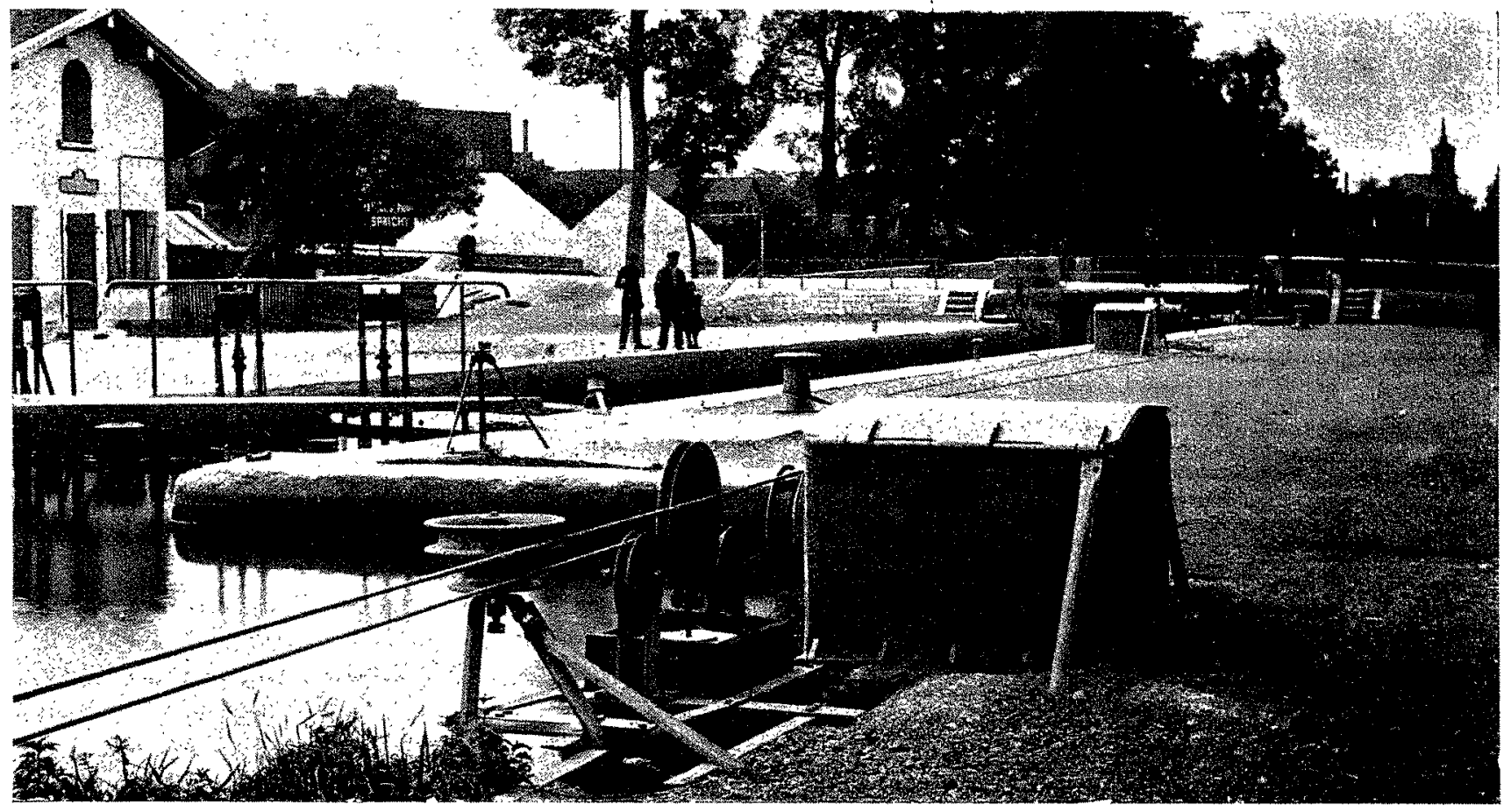

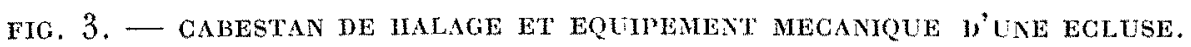

pent qu'un effort insuffisant, aussi les manœuvres se font elles, comme on le sait, avec une très grande lenteur.

Poux accélérer l'éclusage, la Compagnic Générale Electrirue dispose un cabestan dectrique combiné avec des poupécs de renroi placées à chaque exlrémité de l'écluse. Lorsmanouvre du ba-

feau (enlrée et sortie). La durée de manouvre des vannes dépend de la construction même de l'écluse el peul être diminuée par doublement des sas, amenée centrale de l'eau, ete., fous moyens qui nécessitenl de gros travaux de maçonnerie.

la mancuvre du boteau est actuellement fort longue,

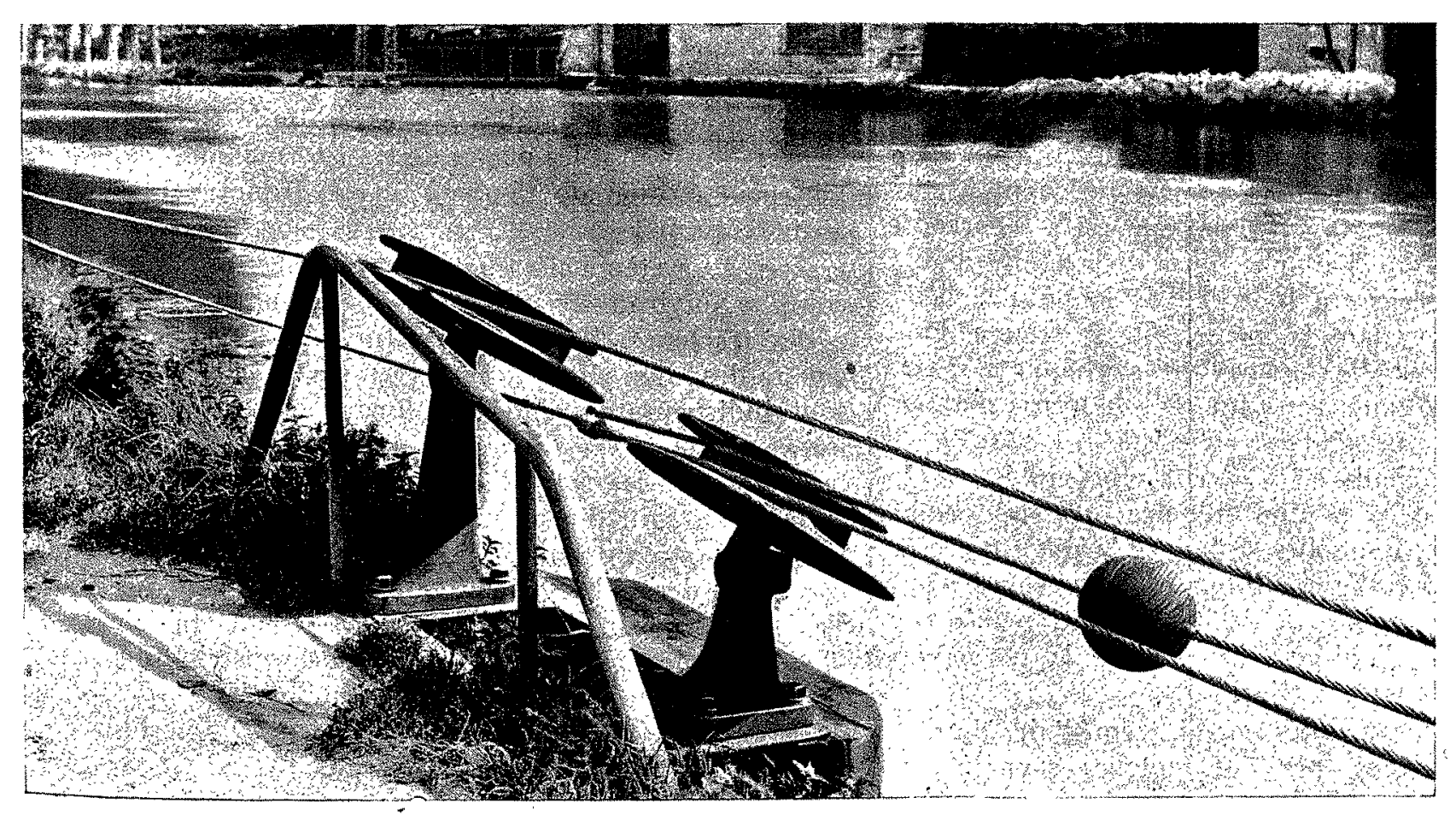

PIC, 4. - POCLIES-GLIDES ET DISPOSTIF D'ACGROCHAGE DE L'AMARIE. qu'um bateau se présente, on l'amarre au moyen d'ume corde qui passe sur la poupée de renvoi siluće à l'extrémilé opposée de l'ćcluse et vient s'enrouler sur la poulie du cabestan. Ce dernier est mis en route, of comme il est susceptible de développer un effort hès élevé, il fait entrer ra-

pilement le baleau dans l'écluse. On l'emploie de la même façon pour la sortie, mais en altachant l'amarre ì l'arrière du batoau (lig. 2.)

Ce disposilif permel de dimjnuer sensiblement In durée d'éclusago. Il est représenté en délail sur la figure 3. Le cabeslan est au second plan el placé vers le milieu de l'c cluse ; aux extrémités on voit les poupées de renvoi. Le cabestan qui rest au premier plan ne fail pas partie du dispositif

car les bateaux que l'on ulilise ont une seclion transversale très peu inférieure à la section ulile de l'écluse. Le bateau, dès qu'il est engagé, forme en quelque sorte piston el l'offort nécessaire pour le faire avancer devient très élevé.

Les chevaux que l'on emploie actuellement ne dévelop- d'éclusage. Examinons mantenant lo détail des appareils.

Descrupton des Appanems

Le càble sans fin est on acier loronné el travaille avec un roefficient de sécurité élevé. Il est actionné par un cabestan 
sur lequel il s'enroule et est supporté, tous les 30 mètres environ, par des poulies de guidage qui le maintiennent.

Un tendeur à poids, placé près du cabestan, raidit le brin mou et assure l'adhérence du câble. Un autre tendeur, réglable à main, est placé à l'autre extrémité et sert à compenser les dilatations et allongements permanents. Sur la figure 3 , on voit, au premier plan, le cabestan de halage, avec, à sa gauche, le tendeur de brin mou. Le poids est placé dans une cheminée qui forme dashpot et amorlit les oscillations violentes.

Laccrochage du câble amarre au cable sans fin présentait deş̧offficultés. Il fallait, en effet, que cette opération soit rendue simple, rapide el, en même temps, très sûre. Il fallait également empêcher le vrillage au point d'attache, afin d'éviter l'enroulement de l'amarre autour du câble sans fin. Il fallait, cnfin, obliger le câble amarre à se dégager sans choc des gorges des poulies de guidage qui supportent le ctble sans fin.

Les dispositifs cmployés par la Compagnie Générale Elecirique pour résoudre ces difficuliés, sont les suivants :

On a placé sur lc câble sans fin, tous les 25 mètres environ, une ligature souple sur laquelle vient s'appuyer un cnsemble de billes en acier et de bagues en bronze enfilées sur le câble. D'autre part, l'amarre du bateau est terminée par une pièce d'accrochage qu'on engage sur le câble et qui vient s'appuyer sur le billage décrit ci-dessus: Get accrochage est combiné de telle façon que la manceuvre en est très simple el très sûre.

Le disposilif décrit ci-dessus ne sert pas seulement à entraîner le bateau, mais il a en outre pour effet de compenser les mouvements de vrillage du câble sans fin. Ce dernicr peul tourner sur lui-mêmo sans difficullé, car, gràce à l'interposition des billes, il n'oblige pas l'amarre à s'enrouler autour de lui.

Le câble sans fin est supporté lous les 30 à 40 mètres par des poulies de guidage. Celles-ci comportent une gorge profonde avec deux joues d'inégale hauleur ; celle côté canal est plus pelite, et, en même temps crénelée d'une façon régulière le long de sa circonférence. Dans son mouvement, le câble sans fin entraîne l'amarre qui vient à côté de lui s'engager dans la gorge. Mais, sur cetle amarre, à environ I mètre du point d'attache, on a disposé une boule de diamètre bien supérieur à la gorge de la poulie. Celle boule ne peut pénétrer dans la gorge, elle se trouve donc soulevée, cl soulève avec elle l'amarre qui s'échappe alors dé la poulie sans que lo cable sans fin ait pu s'en échapper lui-même. (Le fonctionnement est simple).

Les dispositifs précédents se trouvent représentés sur la figure 4 . On voit les deux poulies crénelées qui supportent le câble sans fin, entre lesquelles se trouve la pièce d'accrochage. Celte dernière coiffe les billes et bagues qui s'appuient sur la ligature souple. A droite on voit, placée.sur l'amarre, la boule qui l'obligera à sortir de la gorge des poulie dès qu'elle arrivera sur elles.

Les cabestans sont commandés par l'intermédiaire de plusicurs trains d'engrenages, par des moteurs asynchrones triphasés. Chaque moteur dévcloppe une puissance normale de ro chevaux environ. Afin d'obtenir un matériel aussi robuste que possible, et de réduire au minimum les opérations du démarrage, on emploic des moteurs avec rotor èn court-circuit, ce qui permet de mettre le cabestan en route par la simple mancuvre d'un interrupteur.

Des dispositions spéciales ont été prises pour faire de ces moteurs des organes essentiellement robustes et d'un fonc- lionnement absolument sûr ; ils doivent posséder un couple élevé au démarrage et celui-ci doịt s'effectuer rapidement. En fait, un baleau lourdement chargé est facilement of en un temps très court mis en vitesse. Les mêmes précautions ont été prises pour les moteurs des cabestans de halage dans les écluses dont il a été parlé.

Les moteurs sont alimentés sous une tension de rgo volls. Le courant triphasé est amené le long du chemin de halage par une canalisation à haute tension, la tension étant abaissée à Igo volts dans des postes de transformation répartis d'une façon à peù près uniforme, de manière à alimenter deux cabestans chacun. On a ainsi un poste de transformation avec transformateur statique tous les kilomètres, dans le cas où nous nous sommes placés. Le courant haute tension peut être fourni, suivant les cas, par une usine spéciale ou acheté à des distributions de forces avoisinantes.

Les cabéstans sont commandés, ainsi que nous l'avons vu, par deux interrupteurs d'extrémité à deux positions. Le courant peut d'ailleurs être interrompu entre deux points extrêmes par un nombre; aussi grand qu'on le juge nécessaire d'interrupteurs d'urgence. Pour manœuvrer ces interrupteurs, il faul une clef spéciale que possèdent seuls les conductcurs. Ces interrupleurs permettent d'arrêter le cabestan en cas de besoin, mais ils sont construits de telle façon qu'on ne peut sortir la clef qu'après avoir mis le cabestan en roule. Ce dispositif permet d'éviter toute fausse mancuvre, ne nécéssite-aucun personnel spécial et assure une sécurité absolue de fonclionnement.

\section{Gout de L'Installation}

Si le système de halage par câbles que nous venons de décrire se présente sous une forme simple, robuste, d'un fonctionnement sûr, il a aussi l'avantage de ne pas opposer à sa mise en pratique un prix de revient élcvé et incompatible avec le trafic du canal qui devrait en être pourvu.

Une telle installation nécessile en effet un capital immobilisé relativement faible, représenté par les câbles sans fin, les cabestans et les poulies-guides, la ligne électrique haute et basse tension et les postes de transformateurs, capital que l'on peut évaluer de 55 ooo à 20 ooo francs par kilomètre, au maximum.

Avec le système de tracteurs roulant sur berge, il fant compter environ 25 ooo francs par kilomètre pour la voie et les tracteurs. Il faut, en outre, ajouter à ce chiffre la valcur des sous-stalions de transformation de courant, qui sont indispensables dans la plupart des cas, ainsi que les lignes d'amenée de courant aux tracteurs. Il faut, enfin, tenir compte des frais d'appropriation des berges et, particulièrement, du déplaccment des ouvrages d'art, qui peuvent être extrêmement onércux, sans compter que, dans les traversées des villes, ce système est pour ainsi dire impraticable, en raison des nombreuses lignes de télégraphe, de téléphone, de tramways électrique el de distribution d'énergie, qui croisent les canaux ou longent les berges, créant. de nombreux obstacles a l'étalissement d'une ligne conlinue de traction le long de la berge.

Án point de vue de l'exploitation, le système par cables présente aussi des avantages tròs marqués. La rusticitédu. matériel en rend l'entrelien facile ; la conduite en est simple et n'exige aucun personnel spécial. Enfin, le rendement mécanique est tròs élevé, car l'énergie n'a à subir aucune transformation.

Au conlraire, avec les tracteurs roulants, les locomotives et particulièremént leurs moteurs, nécessitent un entretien 
onéreux. La conduite ne peut être confiée qu’à des wattmen spécialisés. Les sous-stations exigent la présence d'un personnel mécanicien spécial ; quant au rendement, il est fortoment diminué par suite de la transformation qu'on fait subir à l'énergie.

Il faut enfin remarquer avec quelle souplesse le système par câbles sectionnés se prête, non seulement à un trafic intense, mais aussi à un trafic réduit. Quelques bateaux, particulièrement pressés, peuvent sans inconvénient conlinuer à marcher de nuit, ce qui ne serait pas possible avıc un système exigeant l'emploi de sous-stations.

\section{Conclustons}

En résumé, le système de halage par câbles peut être appliqué aux canaux quels que soient leur profil et les ouvrages d'art qui les traversent. Il est simple, robuste et sû́r ; son prix de revient est peu élevé et en pormet la mise en cuvre sur un grand nombre de canaux.

Les essais faits à Jarville, sur lo canal de la Marne au Rhin, et répétés devant de haules personnalités du Corps des Ponts el Chaussées et de l'Industrie, ont donné toute satisfaction et permettent d'affirmer que le système de halago par câble sans fin, que nous venons de décrire, résout. de façon complète ct pratique, le problème de la traction électrique sur les canaux.

\section{STATISTIQUE DE LA NAVIGATION INTÉRIEURE Ex France, anNée r gi I}

Pour compléter les documents qui précèdent, nous reproduisons ci-après, d'après un travail publió dans les annales des Travaux publics de Belgique, le résumé des indicalions fournies par le Ministère des Travaux publics en France. Nous y voyons quel est le remarquable développement de la navigation intérieure et ce scra pour nous un cncouragement à faciliter et à augmenter encore ses moyens d'action.

Tonnace absolu. - Ce tonnage s'est

b́levé pour l'année rgrr à......... 38 rr7648 tonnes.

En rg 10 il n'arait été que de .... $3462379 \mathrm{r}$

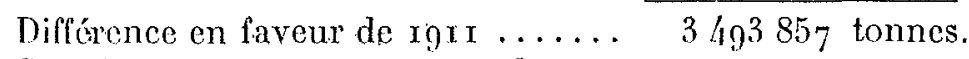
Soil donc une augmentation de ro, i pour 100.

Celte augmentation a porté plus particulièrement sur les transports effectués par les fleuves ol les rivières qui ont gagné rg,4 p. roo; les canaux ont augmenté de 2,6 p. roo.

\begin{tabular}{|c|c|c|}
\hline DESIGNATION DES MARCHANDISES & $\triangle N N B E 1910$ & ANNBE 1911 \\
\hline & Tonnes & Tonnes \\
\hline Combustibles minéraux................ & 11369476 & 12433767 \\
\hline Materiatıx de constmuction, minéranx...... & 11948357 & 13967855 \\
\hline Fingrais ot amendements ............... & 1367790 & 1474101 \\
\hline Bais à hruler et bois de service............ & 1535416 & 1716290 \\
\hline Métaux et Machines...................... & 755413 & 721939 \\
\hline Natières premières de lindris mélallurgique. & 1710461 & 1607971 \\
\hline 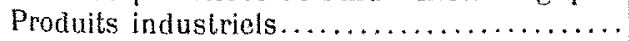 & 1229164 & 1316829 \\
\hline Produits agricoles et denres alimentaires. . & 4.258373 & $439305 i$ \\
\hline Divers $\ldots \ldots \ldots \ldots \ldots \ldots \ldots \ldots \ldots \ldots \ldots$ & 349261 & 362432 \\
\hline Bois flottés de toule espèce............... & 107080 & 124107 \\
\hline Totaux des marchandises tr & 34623791 & 38117648 \\
\hline
\end{tabular}

La répartition par groupes de marchandises, du toinage absolu ayant emprunté l'ensemble des cours d'eau intérieurs français ( ${ }^{\prime}$ ) en $\cdot 19 \mathrm{r}$, ainsi que les quantités correspondantes

(1) Le réseau fréquenté en 1911 a une longueur de 11.354 kilomètres dont 6470 kilomètres pour les rivières et $\$ 881$ kilomilres pour les canaux. de l'année précédente, sont indiquées dans le tableau ci-dessus. Sur 89 cours d'cau ou seclions de cours d'eat, le chiffe de la fréquentation en rgr a dépassé roo ooo tonnes. Sur 57 d'entre cux, le tomnage eflectif s'est élevé au-delà de 500 ono lonnes; sur 35 , il a été supérieur à I million de tonnes, et sur 20 , à 2 millions de tonnes.

Il convient de mentionner particulièrement les cours d'eau dont le tonnage effectif a dépassé 3 millions de tonnes, et dont le développoment total est de 842 kilomètres, ce sont : la Seine, dans la traversée de Paris; la $4^{\circ}$ section de la Seine, comprise entre la limite des départements de Seine-et-Marne et de Seine-et-Oise, el Paris ; la $7^{\circ}$ section, de la Briche a l'Oise ; l'Lscaut, de Cambrai à Elrun ; l'Oise canalisée; la $6^{e}$ section de la Seme, de Paris a la Briche ; la dérivalion de la Scarpe aulour de Douai ; la $S^{e}$ section de la Seine, de l'Oise à Rouen; lo canal de Sant-Quentin; le canal latéral à loise ; la Haute-Deule ; le canal de la Senséc ; lo canal d'Aire et le canal de la Marne au Rhin.

Tonmae knométhipe - Le lonnage ramoné au parcous d'un kilomèlre s'est élevé, en ı̣1, à $5767218 / 60 \mathrm{t}$. k.

L’année précédente, il avait été de.. $5197420130-$

Différence en faveur de 19 I..... 569798330 L. K.

Soit une augmentation de io, 0 pour roo.

Pargolirs moyen d'cne tonne. - 15 i kilomètres en igr et 150 kilomètres en 1910 . (En 1909 , 154 kilomètres.)

RÉPARTTTION DES TRANSPORTS ENTRE LES LIGNES PRINCIPALES ET LES LIGNES SECONDAIIES (1).

Les lignes principales dont le dévoloppement tolal esl de 6 o36 kilomètres, ont reçu 84 pour too du lonnago des marchandises embarquées sur le réseau français el 97 pour roo du trafic kilomélrique lolal ; le tonnage moyen, ramené à la distance entière, de ces lignes ressort à $9277^{3} 7$ lonnes. Il reste seulement, pour les 5.3 8 kilomètres do cours d'eau formant les lignes socondaires, 16 pour roo des embarquements et 3 pour roo du trafic kilomélrique, ce qui réduil à 3 r 477 lonnes leur tomnage moyen.

Le rapprochement de ces chifles montre que la circulalion est environ vingt-neuf fois phus nclive sur les lignes principales, oì s'effectuent les transports ì long cours, que sur los voies secondaires dont le trafic consiste principalement en Iransports locaux.

Il est à noter, enfin, que los voins oi le tonnage absolu a dépassé le chiffre de 5on ooo tomes appartionnent foules aux lignes principales, sauf le canal do f'Oureq, lo canal do Berry, le canal de la Sambre ì l'oise ef le Rhône de lyon à Arles. (Co qui indique le besoin de son aménagement).

Trafic internationar. - Fin rger, co bafic s'est élevé à 4777303 tonnes ; en 19 ro il avait alteint $/ 68 / 4027$ lonnes.

Ce trafic s'est répanti comme suit pour l'exercice y gr :

Enlrées ou imporlalions :

$$
\begin{aligned}
& \text { De Belgique ....... 2 } 3 \text { r } 9690 \text { tonnes. } \\
& \text { D'Allemagne } . . . \ldots \text { 5 5 } 573 \text { - } \\
& \text { Totá...... } 2890 \text { o63 tonnes. }
\end{aligned}
$$

(1) On sait quaux termes de la loi du 5 aout 1879 , relative au classement et a l'amélioration des voies navigables, les voies sont divisés en deux categnries : 10 los lignes principales; 2 Les lignes secondairea.

Le monilage des rivieres el des ranaux constituant les lignes prineipales doit ètre de 2 mètres au minimum ; leurs éluses doivent avoir 5 mo de largeur et $38 m 50$ de longueur ; enfln, pour les canaux, la hauteur libre sous les ponts doit etre d'au moins 3 in 70 . 
Sorties ou exportations :

En Belgique ...... I $3569_{962}$ tonnes.

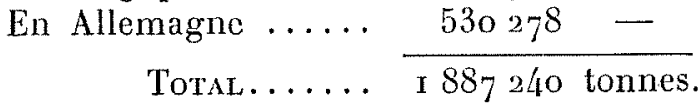

Total du trafic :

Avec la Belgique .... 3676652 tonnes

Avec l'Állemagne ... I $10065 \mathrm{x} \quad$

TотаL...... 4777303 tonnes.

Le tonnage tolal des marchandises importées par cau, en 19 II, présente une augmentation de $107 \mathrm{I}_{7}$ tonnes sur les importations de l'année précédente ; quant aux exportations elles ont diminué de 13 gr I tonnes.

Navig tion à vapeur. - Les statistiques françaises ne rclatent que les marchandises transportées par les batea'ux à vapeur dits porteur's, et ne font pas mention de celles $\mathrm{cm}$ barquées à bord des bateaux ordinaires toués ou remorqués.

Réduite à ces. termes, la part de trafic revenant à la navigation à vapeur est très faible.

Tonmage absolu : 865 o5o lonnes, soit 2,3 pour roo du tonnage total des cmbarquements.

En 1910, le poids transporté avait été de 606268 tonnes; il y a donc eu, en IgIr, une augmentation de $2557 \$_{2}$ tonnes, soit de 42 pour 100.

Tonvage kilométriQue :

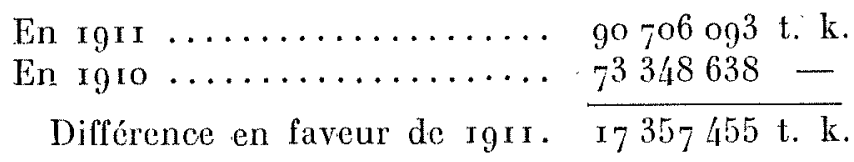

En I9I I, le lonnage kilométrique des batcaux porteurs est égal à $\mathrm{x}, 6$ pour roo du tonnage kilométrique de l'enscmble du réseau (I,4 pour 100, en 1910).

Parcours moyen d'une tonne : io5 kilom. ( 20 en igio).

Mouvement des principaux ports fluviaux. - 652 ports fluviaux (266 pour les rivières et 386 pour les canaux) ont eu, en rgir, un tonnage d'au moins ro ooo tonnes.

Sur ces 652 ports, il y en a : 443 dont le tonnage varie de 10000 à 50 ooo tonnes.

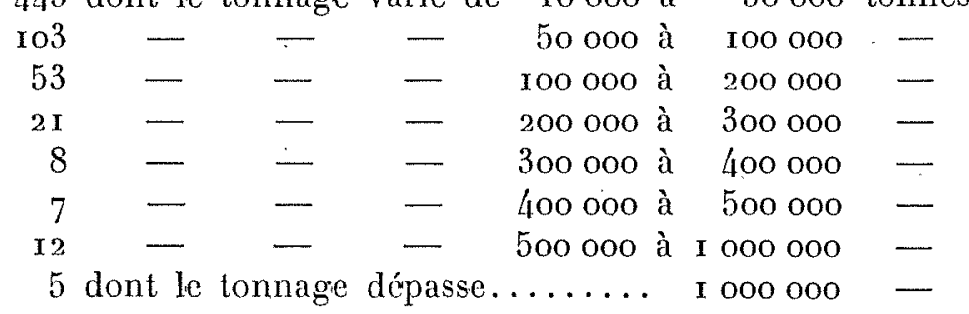

De même que les années antérieures, le port de beaucoup le plus important est celui de Paris, avec un trafic de Io millions 775875 tonnes se décomposant comme suit : embarquements, 3 o2o 895 tonnes; débarquements, 7752980 tonnes. Il y a lieu de remarquer que le mouvement de la navigation dans la traversée de Paris, sur la Seine et les canaux, .est, dans la réalité, plus considérable encore. Il atteint, en y comprenant un trafic local de 346 I 94 tonnes et un transit de r 915 rgo tonnes, le chiffre total de $\times 3$ o35 259 tonnes.

Viennent ensuite Rouen, Vigneux, Villeneuve-le-Roi, Dunkerque et Vendin-le-Vieil, dont le trafic dépasse I million de tonnes ; puis Harnes, Bruay, Bordeaux, Denain, Beuvry, Lyon, Violaines, Montceau-les-Mines, Dombasle, Nanterre, Marles, Lille.

De ces 17 ports, 4 sont situés sur la Seine, I sur la Garonne, I sur le canal du Centre, $x$ sur le canal de la Marne au Rhin, les autres, sauf Lyon, sur les voies du Nord.

\section{SUR LES GOUPS DE BÉLIERS}

DANS LES CONDUITES FORMÉES DE SECTIONS DE DIAMÈTRES DIFFERENTS ( 1 )

Dans les hautes chutes, on est souvent amené à former la conduite de sections dont le diamc̀tre va en diminuant à mesure que l'on s'éloigne de la prise d'eau et que par suite la pression augmente.

On pourrait croire que le coup de bélier ne saurait en aucun cas dépasser la valeur qu'il aurait si la conduite avait partout le diamètre de la partie inférieure, où la vitesse de l'eau est la plus grande. L'élargissement de la parlie supérieure diminuant, en définitive, la force vive lotale de l'eau emmagasinée dans la conduite. Il n'en est toutefois rien, pour une fermeture brusque, ainsi que nous allons le montrer par un exemple simple.

On sait que lorsque la conduite a partout le même diamètre on a, dans le cas d'une fermeture brusque, d'après la théorie de M. Allièvi, pour la valeur du coup de bélier :

$$
\frac{a v_{0}}{g}
$$

où $v_{0}$ est la vitesse de régime, $g$ la gravité et $a$ la vitesse de propagation $\left(^{2}\right)$; de plus, si $l$ désigne la longueur de la conduite, ce coup de bélier se maintient pendant une durće:

$$
\mathrm{T}=\frac{2 l}{a} \text {. }
$$

Il est ensuite suivi d'un coup de bélier négatif de même valeur absolue et de mêrnc durée. Le phénomène présente une série d'oscillations semblables, la durée d'une oscillation complète étant $2 \mathrm{~T}$.

Supposons maintenant une conduite formće de trois sections, la dernière à la partie inférieure de longueur $l$, de diamètre $d$ et où la vitesse de propagation est $a$, les longueurs, diamètres et vitesses de propagation pour les suivantes étant $l^{\prime}, d^{\prime}, a^{\prime}$ ct $l^{\prime \prime}, d^{\prime \prime}, a^{\prime \prime}$, et supposons de plus qu'on ait :

$$
\frac{l}{a}=\frac{l}{a}=\frac{l^{\prime \prime}}{a^{\prime \prime}}
$$

Gonsidérons alors des périodes élémentaires de durée :

$$
\mathrm{T}_{1}=\frac{2 l}{a},
$$

et désignons par $\zeta_{n}$ la valcur du coup de bélicr, pour le cas d'une fermeture totale brusque, pendant la $n^{\text {ieme }}$ période élémentaire, où l'on a, $t$ désignant le temps :

$$
\frac{2(n-1) l}{a}<t<\frac{2 n l}{a} .
$$

Si l'on pose alors :

on aura

$$
\alpha=\frac{a^{\prime} d^{2}}{a d^{\prime 2}}, \quad \beta=\frac{a^{\prime \prime} d^{\prime 2}}{a^{\prime} d^{\prime 2}}, \quad \cos ^{2} \lambda=\frac{\mathrm{I}}{(\mathrm{I}+\alpha)(\mathrm{I}+\beta)},
$$

$$
\zeta_{n}=(-1)^{n-1} \frac{a v_{0}}{g}\left[1-\frac{4 \alpha}{1+\alpha} \frac{\sin n \lambda \sin (n-1) \lambda}{\sin \lambda \sin 2 \lambda}\right]
$$

(1) Note de M. le Comte DE Sparre, à l'A cadémie des Scicnces, séance du 19 mai 1913.

(2) Donnée d'après M. Allièvi par la formule

$$
a=\frac{9900}{\sqrt{48,3+\mathrm{K} \frac{d}{e}}}
$$

où $d$ et $e$ sont lé diamètre et l'épaisseur de la conduite et où $\mathrm{K}=0 ; 5$ pour le fer et l'acier et- $\mathrm{K}=1$ pour la fonte. 